\title{
Efetividade da ozonioterapia em lesões osteonecróticas maxilares - Revisão de
}

\section{Literatura}

\author{
Effectiveness of ozone therapy in maxillary osteonecrotic lesions - Literature review \\ Efectividad de la ozonoterapia en lesiones osteonecróticas maxilares - Revisión de la literatura
}

Recebido: 10/08/2021 | Revisado: 18/08/2021 | Aceito: 21/08/2021 | Publicado: 23/08/2021

\author{
Maria Marina de Mesquita Mourão \\ ORCID: https://orcid.org/0000-0002-5574-8900 \\ São Leopoldo Mandic, Brasil \\ E-mail: marinamourãom@gmail.com \\ Yrio Lemos \\ ORCID: https://orcid.org/0000-0002-5525-3377 \\ Faculdade Meta, Brasil \\ E-mail: yriolemos@unimeya.edu.br \\ Angela Nascimento Carvalho \\ ORCID: https://orcid.org/0000-0001-6186-3350 \\ São Leopoldo Mandic, Brasil \\ E-mail: profangelaodonto@gmail.com \\ Maria Aparecida Rodrigues de Holanda \\ ORCID: https://orcid.org/0000-0002-8797-2720 \\ Universidade Estadual Paulista, Brasil \\ E-mail: cida_holanda@outlook.com.br \\ Kleyton Nolasco de Abreu \\ ORCID: https://orcid.org/0000-0001-9035-9268 \\ Centro Universitário Uninorte, Brasil \\ E-mail: kleytonnolasco@hotmail.com \\ Elisa Rebeca de Holanda Balestra \\ ORCID: https://orcid.org/0000-0002-0173-8707 \\ Faculdade de Odontologia de Manaus, Brasil \\ E-mail: elisa.rebeca@hotmail.com \\ Samuel Barbosa Macedo \\ ORCID: https://orcid.org/0000-0002-5445-4601 \\ Universidade Federal de Rondônia. Brasil \\ samuelbamacedo@hotmail.com \\ Ellen Roberta Lima Bessa \\ ORCID: https://orcid.org/0000-0002-1178-2568 \\ Universidade Estadual Paulista, Brasil \\ E-mail: ellenrlb@hotmail.com \\ Ana Bessa Muniz \\ ORCID: https://orcid.org/0000-0003-4414-9854 \\ Universidade Estadual Paulista, Brasil \\ E-mail: bessa.muniz@unesp.br
}

\section{Resumo}

A utilização do ozônio na odontologia tem sido indicada devido as suas propriedades. Estas o tornaram um importante adjuvante no manejo de diversas situações odontológicas. O mecanismo de ação faz da ozonioterapia uma técnica com diversos atrativos para as especialidades odontológicas. A estomatologia tem se beneficiado com os estudos sobre a temática, em especial, o manejo de lesões osteonecróticas dos maxilares. Através de uma revisão de literatura, o objetivo deste trabalho é resumir as diferentes possibilidades da utilização do ozônio, dentro das especialidades odontológicas, destacando sua aplicabilidade na prevenção e tratamento de osteonecrose dos maxilares. Foi observado que a ozonioterapia se mostrou uma ferramenta versátil e eficaz, se comportando como um auxiliador do sistema imunológico favorecendo o reparo de enfermidades odontológicas com caráter crônico e/ou agudo. O ozônio também se mostrou um potente redutor de complicações, quando se tratando de pacientes com risco de desenvolvimento de osteonecrose, além de contribuir para a cura de lesões já estabelecidas.

Palavras-chave: Ozonioterapia; Osteonecrose de maxilares; Odontologia.

\section{Abstract}

The use of ozone in dentistry has been indicated due to its properties. These have made it an important adjunct in the management of various dental situations. The mechanism of action makes ozone therapy a technique with several attractions for dental specialties. Stomatology has benefited from studies on the subject, especially the management of osteonecrotic lesions of the jaws. Through a literature review, the objective of this work is to summarize the different 
possibilities of using ozone within dental specialties, highlighting its applicability in the prevention and treatment of osteonecrosis of the jaws. It was observed that ozone therapy proved to be a versatile and effective tool, behaving as an immune system helper, favoring the repair of dental diseases with a chronic and / or acute character. Ozone has also been shown to be a potent reducer of complications when it comes to patients at risk of developing osteonecrosis, in addition to contributing to the healing of already established injuries.

Keywords: Ozone therapy; Jaw osteonecrosis; Dentistry.

\section{Resumen}

Se ha indicado el uso de ozono en odontología por sus propiedades. Estos lo convirtieron en un adyuvante importante en el manejo de diversas situaciones dentales. El mecanismo de acción hace de la ozonoterapia una técnica con varios atractivos para las especialidades dentales. La estomatología se ha beneficiado de estudios sobre el tema, en particular, el manejo de las lesiones osteonecróticas de los maxilares. Mediante una revisión de la literatura, el objetivo de este trabajo es resumir las diferentes posibilidades de uso del ozono, dentro de las especialidades dentales, destacando su aplicabilidad en la prevención y tratamiento de la osteonecrosis de los maxilares. Se observó que la ozonoterapia resultó ser una herramienta versátil y eficaz, actuando como ayuda al sistema inmunológico, favoreciendo la reparación de enfermedades dentales crónicas y / o agudas. También se ha demostrado que el ozono es un potente reductor de complicaciones cuando se trata de pacientes con riesgo de desarrollar osteonecrosis, además de contribuir a la curación de lesiones ya establecidas.

Palabras clave: Ozonoterapia; Osteonecrosis mandibular; Odontología.

\section{Introdução}

O Ozônio $\left(\mathrm{O}_{3}\right)$ é um composto químico natural triatômico, constituído por três átomos de oxigênio, encontrado naturalmente na forma de gás na camada superior da atmosfera em concentrações de 1- 10 ppm (partes por milhão). É um dos gases mais importantes na manutenção do equilíbrio na biosfera, pois protege os seres vivos contra a radiação ultravioleta (Ferreira, 2011). Ele representa o segundo elemento com maior poder oxidativo, e sua formação (Fig. 1) se dá a partir de uma descarga elétrica sobre a molécula de oxigênio, a qual tem suas ligações quebradas liberando seus átomos (Naik et al., 2016). Estes, por sua vez, se unem a outras moléculas de oxigênio, originando o $\mathrm{O}_{3}$ (Gopalakrishnan \& Parthiban, 2012). Por ser extremamente reativo, estabelece reação com qualquer poluente que entre em contato com muita facilidade e, devido a sua instabilidade, ele retorna com facilidade para forma molecular original de oxigênio (Tiwari et al., 2016).

Figura 1 - Formação do Ozônio.

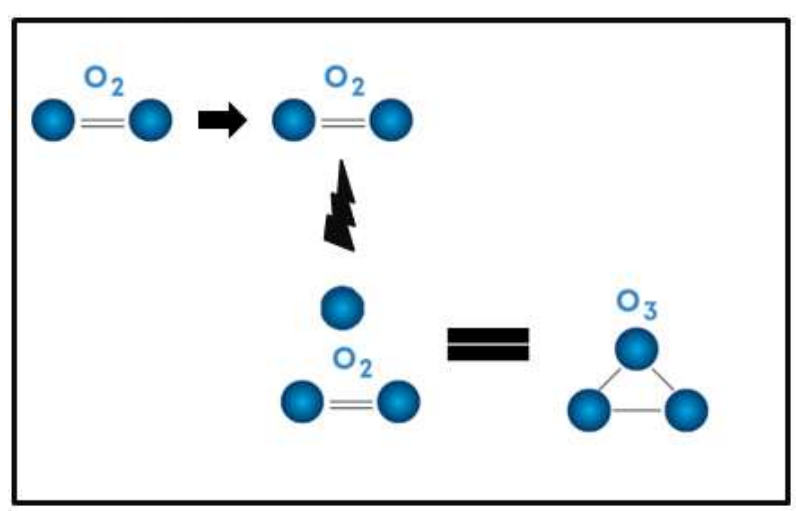

Legenda: Molécula de Oxigênio sofrendo descarga elétrica, átomo de $\mathrm{O}$ fazendo ligação com molécula de $\mathrm{O}_{2}$ resultando em $\mathrm{O}_{3}$. Fonte: Autores.

O ozônio é um gás instável que deve ser utilizado logo após a sua sintetização, sendo sua meia-vida influenciada pela temperatura, onde temperaturas mais baixas conseguem manter sua concentração por períodos mais prolongados. $\mathrm{O} \mathrm{O}_{3}$ possui um elevado potencial oxidativo eficaz na destruição de bactérias, fungos, vírus e protozoários além de ser mais denso e solúvel na água quando comparado ao oxigênio, o que aumenta a sua capacidade de penetração nos tecidos e dissolução no plasma sanguíneo (Ali et al., 2018; Ferreira, 2011). 
Neste contexto, a ozonioterapia é uma terapia bio-oxidativa, que tem ação em nível celular, é utilizada como método terapêutico auxiliar de quadros patológicos infecciosos e/ou inflamatórios de caráter agudo ou crônico (Ferreira, 2011). Seu modo de administração na odontologia é tópico, através de três formas básicas de aplicações; 1) gás ozônio; 2) água ozonizada; 3) óleo ozonizado. Para a sua sintetização e utilização como agente terapêutico, é necessário a passagem de oxigênio puro pelo gerador de ozônio medicinal, tendo como produto final, a mistura gasosa de $95 \%$ de oxigênio e $5 \%$ de ozônio, concentração menor do que aquela usada para fins industriais (Nesi, 2018).

São diversas as propriedades do $\mathrm{O}_{3}$, porém, algumas têm se destacado no uso odontológico, como: ação antimicrobiana (Huth et al., 2005; Huth et al., 2006; Huth et al., 2009) atuando sobre os ácidos graxos poli-insaturados da membrana celular; poder bio-estimulante, influenciando a produção de células imunocopetentes e imunoglobulinas; ação antioxidante através da liberação de substratos biologicamente ativos; ação antiflamatória e analgésica pela baixa na produção de mediadores da inflamação (Naik et al., 2016; Smith et al., 2017). Outro efeito abordado na literatura da Ozonioterapia, é sua ação revitalizante e produtora de energia, restaurando o metabolismo do oxigênio e reativando alguns ciclos enzimáticos (Luis et al., 2013).

$\mathrm{Na}$ literatura, diversos estudos comprovam a versatilidade do ozônio nas mais variadas especialidades odontológicas (Pulga et al., 2018; Suh et al., 2019). Na dentística, para desinfecção de lesões cariosas e na dessensibilização dentinária. Na endodontia, sendo utilizado como irrigante, medicação intra-canal e como agente clareador de elementos desvitalizado nas áreas cirúrgicas (Nogales, 2011, Reddy et al., 2013; Nogales et al., 2016; Nogales et al., 2020). Ademais, na área da periodontia, o ozônio acelera a cicatrização, estimula o reparo ósseo e auxilia nos processos desinfetante, e a sua aplicação de forma tópica atua como antimicrobiano local (Gupta \& Mansi, 2012, Srikanth et al., 2013). Além disso, atua desde da cicatrização de lesões em tecido mole como herpes, ulceras traumáticas até na reversão de distúrbios da articulação temporomandibular (Garg \& Tandon, 2009; Nesi, 2018; Naik et al., 2016; Gopalakrishnan \& Parthiban, 2012; Ferreira, 2011).

Por décadas, o ozônio tem sido usado como alternativa para tratamento de diversos quadros patológicos na odontologia, entre eles, a osteonecrose dos maxilares (ONM). Com frequência é observado que estes pacientes, não respondem de forma positiva apenas à antibioticoterapia e aos cuidados tradicionais. Atualmente, muitas pesquisas avaliaram a ozonioterapia como alternativa para a resolutividade desse quadro em consequência de seus efeitos biológicos, sendo apontado como uma alternativa favorável, não invasiva, e de alta efetividade para a ONM (Fliefel et al., 2015; Agrillo et al., 2007a).

Este trabalho tem como objetivo avaliar a efetividade da ozonioterapia na odontologia, e sua eficácia no tratamento e prevenção de osteonecrose dos maxilares, através de uma revisão da literatura.

\section{Materiais e Métodos}

A presente revisão da literatura integrativa foi elaborada através de uma análise narrativa conforme as orientações Botelho et al. (2011), iniciando-se com uma breve contextualização sobre o uso do ozônio na medicina humana e na odontologia, especificamente a sua utilização no tratamento de osteonecrose. Esse método de revisão permite a revisão de métodos, teorias, e/ou estudos empíricos sobre um tópico particular, o que permite tecer a partir de estudos já realizados, um novo conhecimento. A metodologia tem como principal vantagem, em relação à revisão puramente narrativa, o maior rigor metodológico, além de ser mais específica e delimitada em relação ao tema abordado. O referencial teórico foi confeccionado por meio da revisão da literatura científica publicada em periódicos, nas bases de dados online PubMed, Lilacs e Ebsco, acessados por meio de ferramentas de busca na internet, tanto em linguagem nacional, quanto internacional. Foi realizada uma leitura inicial dos títulos e resumos, afim de selecionar material para posterior contemplação. Foi selecionado um total de 37 arquivos publicados entre 2005-2020, composto por 32 artigos e 5 trabalhos de conclusão de curso, incluindo dissertações e teses. Foram abordados trabalhos quantitativos diretamente relacionados com o uso da ozonioterapia em casos de osteonecrose, 
como também foram utilizados artigos complementares para melhor discussão e compreensão do tema. As palavras chaves utilizadas para filtrar os resultados de busca foram Ozonioterapia, Osteonecrose de Maxilares e Odontologia.

\section{Resultados}

Inicialmente, a osteonecrose foi associada a pacientes submetidos à radioterapia de cabeça e pescoço, e foi conhecida como Osteorradionecrose (ORN). Posteriormente, em osteonecrose dos maxilares, foi relacionada ao uso de determinados medicamentos (ONMRM) (Ellis, 2015; Ribeiro et al., 2018), em especial os remodeladores ósseos e antiangiogênicos.

$\mathrm{O}$ aparecimento do quadro é resultante de um conjunto de fatores internos e/ou externos (radiação, drogas, exposição à químicos), podendo ser desencadeados por infecção, extração dentária, trauma mecânico nas maxilas ou o uso concomitantemente de drogas imunossupressoras, que podem causar desequilíbrio na homeostase mineral. $\mathrm{O}$ resultado dessa desregulação na capacidade de remodelação óssea pode levar à perda de tecido ósseo como na osteonecrose (Omolehinwa \& Akintoye, 2016; Ribeiro et al., 2018; Ripamonti et al., 2011). Devido a sua alta densidade mineral e menor suprimento, a mandíbula é o osso mais comumente afetado pelas osteonecroses de cabeça e pescoço (Ellis, 2015).

A osteonecrose dos maxilares tem sido apontada como um dos principais efeitos colaterais ao uso dos bifosfonatos (BFs), substâncias análogas sintéticas do pirofosfato inorgânico (Agrillo et al., 2007a; Fliefel et al., 2015; Ikebe, 2012), que é um regulador natural da calcificação e inibidor da reabsorção óssea, presente no organismo (Ribeiro et al., 2018; Agrillo et al., 2012). Estas drogas, são comumente utilizadas para o tratamento de doenças envolvendo reabsorção óssea. Sua meia vida é aproximadamente 10 anos, isso é resultado de sua composição química, onde encontra-se o grupo hidroxila que possui uma forte afinidade à hidroxiapatita, com isso, $80 \%$ do BFs administrado ficam depositados nos ossos. O seu mecanismo de ação envolve a inibição do processo de reabsorção, diminuindo a ativação dos osteoclastos e induzindo a sua apoptose, e inibindo a atividade da bomba de prótons osteoclástica (Fliefel et al., 2015; Agrillo et al., 2007b).

Estima-se que em média 70\% dos efeitos colaterais dos bifosfonatos são clinicamente indetectáveis até algum evento traumático desencadeante, como: infecções, extração dentária, trauma mecânico nas maxilas ou pelo uso contínuo de drogas imunossupressoras. Enquanto 30\% dos casos restantes de ONMRM, ocorrem em um estágio inicial como consequência direta da ação dos BFs sem qualquer efeito desencadeante plausível (Fliefel et al., 2015; Agrillo et al., 2007a; Agrillo et al.2012).

Além do uso dos BFs, a osteonecrose pode se formar em decorrência a radiação de cabeça e pescoço (Batinjan et al., 2014). Quando a causa é a radiação, ocorre a destruição da microvascularização, o osso irradiado permanece com a taxa de renovação baixa tornando o processo de autorreparação ineficiente (Ellis, 2015), sendo que a radiação instala um processo inflamatório levando à apostose dos osteoblastos (Ribeiro et al., 2018).

As manifestações clinicas dependem do curso da doença (Ribeiro et al., 2018). Clinicamente, a doença se caracteriza por exposição de osso necrótico persistente, por pelo menos três meses; capaz de manifestar-se sem área óssea exposta com bolsa periodontal profunda; apresentar elemento dentário com mobilidade, trismo, hipoestesia do lábio inferior e dor de origem não odontogênica; inchaço em tecido moles, dor difusa com presença de fistulação; dor à mastigação, comprometimento vocal (Omolehinwa \& Akintoye, 2016; Ribeiro et al., 2018; Fliefel et al., 2015; Agrillo et al., 2012).

O tecido ósseo degenerativo fornece um ambiente ideal para a colonização bacteriana e, devido a cavidade oral ser constituída por um ambiente polimicrobiano, é comum o surgimento de superinfecções nos casos de osteonecrose maxilares (Agrillo et al., 2007a ; (Ferreira et al., 2013).

As recomendações clinicas de tratamento e/ou prevenção das osteonecroses são várias e incluem medidas como antibioticoterapia, enxague com solução de clorexidina, debridamento cirúrgico, remoção cirúrgica da lesão ou a administração de oxigênio hiperbárico. Porém, a desregulação da homeostasia óssea, afeta a efetividade das medidas adotadas (Ripamonti et 
al., 2011; Agrillo et al., 2007b; Fliefel et al., 2015). Quando fala-se de osteorradionecroses, algumas terapias preventivas incluem administração de dois medicamentos, a pentoxifilina e o tocoferol (Ribeiro et al., 2018).

Atualmente, o número de pesquisas avaliando a ozonioterapia como alternativa para a resolutividade desse quadro vem aumentando. Esta é reconhecida por vários Sistemas de Saúde no mundo, gradativamente estão sendo adicionados aos hospitais públicos, sendo muito utilizada como um complemento nas áreas oncológicas, visando à diminuição dos efeitos colaterais da radioterapia (Alves, 2017).

Segundo Agrillo et al. (2012), o conceito para utilizar esta terapia nos casos de ONM, é que, o ozônio melhora o quadro geral da lesão, para que procedimentos cirúrgicos minimamente invasivos possam ser realizados sem complicações. Agrillo et al. (2007a) mostraram o desfecho da situação clínica de 15 pacientes submetidos à um ciclo de ozonioterapia pré e pós cirúrgico, juntamente com antibioticoterapia (sete dias antes e sete dias após), que estavam em tratamento com bifosfonatos. Nas vinte extrações realizadas, não foi relatada nenhuma complicação. Estes resultados mostraram que há possibilidade de extração nesta parcela de pacientes, após aplicação de pelo menos um ciclo prévio de ozônio.

Agrillo et al. (2007b) realizaram um experimento clinico envolvendo pacientes acometidos por ONMRM e tratamento com ozonioterapia. Foram incluídos 33 pacientes que passaram por cirurgias não invasivas e ciclos pré e pós cirúrgicos de ozonioterapia. Foram realizadas oito sessões de três minutos cada, além de terapias antibióticas e antifúngicas. Foi observada cicatrização completa em 54\% dos pacientes e redução clara do tamanho das lesões e melhora de sintomatologia em $30 \%$. Apenas em 16\%, apesar terem relatados melhoras na sintomatologia, não houveram alterações relevantes. Isso evidencia que a ozonioterapia potencializa os benefícios dos tratamentos cirúrgico e farmacológico, contribuindo para a cicatrização das lesões e melhora dos sintomas e inibição da progressão das lesões. Os autores concluíram que a ozonioterapia é previsível e confiável no tratamento de ONMRM.

Em um estudo, Ripamonti et al. (2011) apresentaram uma opção terapêutica promissora, analisando o efeito de óleo ozonizado no tratamento de dez pacientes acometidos por osteonecrose associados ao uso de BFs que não responderam ao tratamento com antibióticos. Todos os pacientes foram submetidos à antibioticoterapia prévia por dez dias antes da aplicação do ozônio, e tartarectomia. O óleo ozonizado foi aplicado diretamente nas lesões, com máximo de dez aplicações a cada três dias, dez minutos por sessão. Houve acompanhamento pós-tratamento de até 8 meses e considerou-se sucesso clínico quando houve: sequestro total ou parcial de osso necrótico; cicatrização e reepitelização da mucosa com tecido epitelial regenerado. Todos os casos alcançaram uma boa resolução do quadro, levando em média 27 dias de tratamento 80\% dos pacientes apresentaram expulsão espontânea de fragmentos necróticos e $20 \%$ desenvolveram a formação de novos ossos em torno da área necrótica seguidos de reepitelização da mucosa.

Agrillo et al. (2012) realizaram um estudo retrospectivo realizado entre 2004 a 2010, envolvendo 94 pacientes afetados por ONM, avaliram a dor pré- e pós-ozonioterapia. Foi realizado a remoção dos sequestros ósseos e duas sessões de ozonioterapia por semana, com duração de três minutos, em conjunto como antibioticoterapia e terapia anti-fungica, e administração de ácido ascórbico, juntamente com bochecho de clorexidina à $0,2 \%$ até a resolução completa do quadro. A dor após curetagem da lesão óssea foi de $27,6 \%$ e $0 \%$ no pré- e pós-operatória, respectivamente, indicando que a ozonioterapia tem efeito previsível na dor. Mostrou-se, também, que 60\% dos casos obtiveram redução completa dos sintomas; $30 \%$ uma redução acentuada das lesões, com melhora nos sintomas; e 10\% chegaram a uma cura parcial sem alteração na lesão, resultados bem-sucedidos em $90 \%$ dos casos.

Ripamonti et al. (2012) em um estudo prospectivo em 24 pacientes com osteonecrose maxilar medicamentosa, avaliaram a eficácia e tolerabilidade do gás ozônio no tratamento destas lesões e concluíram que esse tipo de terapia é seguro e eficaz, principalmente em pacientes com lesões maiores que $2,5 \mathrm{~cm}$. No referido estudo, os indivíduos receberam insuflação tópica com $\mathrm{O}_{3}$ a cada três dias, com no mínimo 10 aplicações para cada área patológica, ou até a remoção do osso necrótico de 
forma espontânea ou cirúrgica. Foi realizada antibioticoterapia concomitante com azitromicina. 11 dos 24 pacientes necessitaram de mais 10 insuflações de gás $\mathrm{O}_{3}$. Seis pacientes interromperam a terapia por progressão da doença oncológica, e seis pacientes apresentaram sequestro e expulsão espontânea completa ou parcial do osso necrótico, seguida de reepitelização da mucosa oral. Não houve eventos adversos. Em 12 pacientes com as maiores lesões, o ozônio produziu o sequestro do osso necrótico após 10 a 38 insuflações.

Para saber sobre os tratamentos disponíveis para ONMRM, e seus resultados, os autores Fliefel et al. (2015) desenvolveram uma revisão sistemática contemplando 97 artigos publicados de 2003 à 2014. Foi observado que, o tratamento cirúrgico minimamente invasivo e tratamento médico, foram os mais utilizados. Dentre os adjuvantes incluíram laser, fatores de crescimento, oxigênio hiperbárico e ozônio. Os artigos forneceram uma ampla gama de variáveis de resultado para avaliar o tratamento de ONMRM e os resultados de um. Dentre os 161 pacientes tratados com ozônio nos artigos publicados, 57,85\% apresentaram cura completa, 16,8\% mostraram cicatrização parcial e 3,1\% apresentaram lesões estáveis; 17,4\% tiveram lesões regressivas, $5 \%$ não apresentaram cura e nenhum paciente apresentou lesões progressivas ou recorrentes.

Batinjan et al. (2014), por meio de um relato de caso de um paciente de 55 anos submetido à radioterapia de cabeça e pescoço, apresentaram a ozonioterapia como opção para prevenção e tratamento de osteorradionecrose. Foram constatadas durante o exame clínico candidíase e mucosite, causados por xerostomia severa, além de raízes residuais inferiores e superiores, parcialmente sem vitalidade pulpar. O protocolo proposto envolveu a extração de todos os elementos superiores, com aplicação preventiva de ozônio para redução da carga microbiana, e após a extração, uma nova aplicação de ozônio dentro do alvéolo. Uma terceira aplicação foi realizado após a sutura com intuito de estimular a circulação e cicatrização. Os resultados mostraram a ausência de sinais indicativos de ORN, ausência de complicações nas cicatrizações, evidenciando um efeito positivo da ozonioterapia na prevenção e cicatrização de múltiplas feridas em paciente de alto risco.

Outro relato de caso de Rodrigues (2016) comprovou a efetividade da ozonioterapia no tratamento de uma paciente acometida por osteonecrose mandibular. A paciente fazia uso de $60 \mathrm{mg}$ de antirreabsortivo injetável para o tratamento de osteoporose pós-menopausa. Um ano após o início do tratamento, foram realizadas extrações dos molares inferiores, induzindo ao desenvolvimento da osteonecrose, com gengiva integra sem exposição óssea, parestesia de nervo mentoniano do lado direto, e presença de fistula extraoral na região submentoniana, originada da área necrótica correspondente à região dos elementos 44 e 45. Devido à integridade do tecido gengival, as aplicações de ozônio ao osso necrótico foram realizadas via canal do elemento 44, com ozônio gasoso e aquoso, em concentrações maiores para promoção de debridamento químico da necrose. As demais aplicações de ozônio por visarem à estimulação tecidual intra-lesional, tiveram suas concentrações reduzidas. Foram realizados também aplicações no fundo de sulco para estimulação de resposta tecidual perilesional. Já após três primeiras aplicações de ozônio, foi notado o início da ressensibilização na região, na quinta aplicação, houve sangramento via canal indicando o início da revascularização óssea. Após a finalização das 21 e 12 aplicações externas e internas, respectivamente, foi observado a remissão da inflamação, e recuperação da sensibilidade, além de total cicatrização da fistula extra oral.

Através de um relato clinico, Alves (2017) demonstrou a aplicabilidade da ozonioterapia na osteonecrose dos maxilares relacionados à medicamentos e sua implicância clínica. No presente relato, a paciente fez uso de Alendronato de sódio (BFs) por via oral durante cinco anos. No exame físico, foi detectada exposição óssea necrótica na região anterior do rebordo alveolar e região perimplantar, com formação de abscesso na região submandibular. Foi utilizado a ozonioterapia em duas formas tópicas, água e óleo ozonizados. O debridamento ósseo, desepitelização e remoção de sequestro ósseo foram realizados. Observou-se o fechamento não cirúrgico em região de exposição óssea necrótica, sem nenhum efeito colateral ou prejuízo ao paciente.

Akdeniz et al. (2018), afim de investigar o efeito do gás ozônio na cicatrização de feridas em fibroblastos humanos afetados pelo uso de BFs, cultivaram, in vitro, fibroblastos gengivais, os quais receberam concentrações citotóxicas de 
bifosfonatos com protocolo de aplicação de plasma de gás ozônio na concentração de $60 \mu \mathrm{g} / \mathrm{mL}$ por 30 segundos. Os resultados mostraram que a aplicação do ozônio diminuiu significativamente o dano genotóxico, além de aumentar as taxas de cicatrização.

\section{Discussão}

Embora a utilização do ozônio nas áreas da saúde tenha se intensificado desde a sua aplicação pioneira na primeira guerra mundial, Sabbah et al. (2018) afirmam que o $\mathrm{O}_{3}$ ainda não é considerado uma modalidade de tratamento, possivelmente tal condição seja explicada pela escassez de fundos destinados a pesquisa pública e pelo desinteresse das empresas farmacêuticas em modalidades não patenteáveis (Gopalakrishnan \& Parthiban, 2012; Sabbah et al., 2018). Nogales et al. (2008) ressaltam que embora o interesse pelo ozônio venha crescendo juntamente com pesquisas voltadas em determinar diretrizes em sua utilização, é notável a discrepância sobre a duração de tempo que as aplicações de ozônio devem ter.

Apesar da liberação da FDA (Food and Drug Administration) para a utilização do ozônio como antimicrobiano de alimentos desde 2001, o Conselho Federal de Odontologia (CFO) só o reconheceu em 2015, por meio da resolução $\mathrm{n}^{\circ}$ 166/2015. Sabbah et al. (2018) verificou em seu trabalho que dentre as publicações, o Brasil encontra-se como o quinto pais onde estão concentrados o maior número de publicações sobre a ozonioterapia.

Além da atuação do ozônio bem sedimentada nas especialidades odontológicas, é encontrado na literatura que a ozonioterapia é reconhecida como uma técnica segura quando observada suas normas e restrições. Apesar de complicações não ocorrerem com frequência, alguns aspectos durante o manejo do ozônio devem ser respeitados. Segundo Swanson e Chapman (2019), aspectos como a concentração, localização e duração da exposição ao ozônio devem ser avaliados cuidadosamente.

Os autores Agrillo et al. (2007b), Ripamonti et al. (2011) e Fliefel et al. (2015) manifestam pontos de vista similares em relação a ozonioterapia no tratamento de ONMRM. Para eles, as propriedades oferecidas pelo $\mathrm{O}_{3}$ se alinham para a resolução de uma grande maioria das manifestações clínicas apresentadas pela osteonecrose. Os autores explicam que o ozônio, quando atuando na osteonecrose, tem o propósito de restaurar a fisiologia normal do osso ao manter o sistema antioxidante, estimular o sistema circulatório e o metabolismo de minerais. Em consequência disso, as pesquisas avaliando a ozonioterapia como alternativa para a resolutividade desse quadro vem aumentando.

É perceptível o crescente número dos casos de osteonecrose desde a primeira menção na literatura. O desenvolvimento desta doença pode ser afetado por alguns fatores ligados aos bifosfonatos, como duração do tratamento, dose cumulativa e o tipo de BFs utilizado. Segundo Fliefel et al. (2015), bifosfonatos contendo azoto como o Zoledronato e Pamidronato impelem risco mais elevado para o desenvolvimento da patologia. Do mesmo modo, um dos resultados obtidos por Akdeniz et al. (2018), revelou que, Zoledronato e o Pamidronato causaram maior dano genotóxico às células de fibroblastos gengivais humanos. Isto pode ser explicado pelo efeito inibitório, taxa de rotatividade e atividade antirreabsortiva mais potentes nesses tipos de BFs.

Segundo o trabalho exposto por Agrillo et al. (2007a), a capacidade de estimular e preservar os antioxidantes endógenos nas proximidades da aplicação do ozônio e não apenas localmente, diminuem os riscos de possíveis complicações nos casos de extrações dentárias em pacientes que estão utilizando bifosfonatos. Este fato pode ser comprovado em seu estudo, no qual 15 pacientes sob uso de bifosfonatos, receberam aplicações de ozônio e mais tarde sofreram extrações dentárias, não relataram quaisquer tipos de complicações ósseas.

É observado na literatura que embora o ozônio apresente resultados favoráveis, essa modalidade de tratamento ainda não é a primeira escolha diante das osteonecroses (Agrillo et al., 2007a). Fliefel et al. (2015) por meio de uma revisão sistemática, comprova isto, revelando que dentre os 3475 pacientes encontrados nas pesquisas publicadas, apenas 4,6\% foram tratados com ozônio. Tais resultados são corroborados pelo trabalho de Ribeiro et al. (2018), que analisaram a literatura 
publicada nos últimos 11 anos sobre as possíveis terapias para osteorradionecrose, onde foi observado que a grande maioria das publicações incluíram a antibioticoterapia isolada ou combinada como tratamento.

A presença do antibiótico sistêmico é importante, principalmente quando há associação de infecções secundárias agudas e para prevenir a disseminação dessas infecções. Ellis (2015) especifica que as osteonecroses não são infecções ósseas causadas por microorganismos, mas sim um ferimento agravado pela hipóxia tecidual que não cicatrizaram. Sendo assim, a diminuição da vascularizaçã o deve ser levada em conta, visto que dificultará a chegada dos antibióticos às áreas lesadas.

É perceptível que a opção pelo ozônio ocorre na maioria dos casos quando outras alternativas de tratamento anteriores não foram bem-sucedidas. O que pode ser constatado no estudo de Ripamonti et al. (2012), onde apresenta o efeito da ozonioterapia em casos de osteonecrose já tratados anteriormente por alguma terapia que apresentou insucesso. Neste estudo, os resultados com as insuflações de ozônio se mostraram positivas, resultando na possibilidade de remoção dos sequestros ósseos sem maiores complicações. Foi notado, também, a cicatrização das lesões necróticas mais profundas. Os autores atribuem esses resultados ao potencial oxidante do ozônio, que não só destrói a membrana de microrganismos, como também desencadeia uma fase reparadora levando à cicatrização dos tecidos lesados. Resultados semelhantes foram obtidos por Ripamonti et al. (2011) em outro estudo, apesar da ozonioterapia ter sido aplicada na sua forma oleosa. Nesta pesquisa $80 \%$ dos pacientes apresentaram expulsão espontânea dos fragmentos necróticos e $20 \%$ desenvolveram a formação de novos ossos em torno da área necrótica seguidos de reepitelização da mucosa.

Agrillo et al. (2012) mencionam que o uso da ozonioterapia nas osteonecroses é baseada na ideia de que o ozônio irá melhorar as condições locais possibilitando a realização de tratamentos cirúrgicos minimamente invasivos. A metodologia aplicada neste estudo envolveu remoção dos sequestros ósseos, juntamente com antibióticos sistêmicos associados à ozonioterapia. Um dos resultados mostraram que o ozônio possui efeito positivo sobre a sintomatologia das lesões, devido à modulação que promove nas células imunológicas, como também a estimulação de angiogênese e formação de fibroblastos. Em conformidade com o resultado anterior, outros estudos, mostraram que, além da cicatrização completa das lesões (54\%), houve redução significativa na sintomatologia (46\%) após as aplicações de ozônio pré e pós-cirurgia não invasiva (Agrillo et al., 2007b).

\section{Conclusão}

Com os estudos expostos, é observado que a ozonioterapia se mostrou uma ferramenta eficaz na prática odontológica, trazendo consigo inúmeras possibilidades de aplicações na área da saúde. Além de seu excelente poder antimicrobiano e sua biocompatibilidade, o ozônio apresenta atuação direta no sistema imunológico contribuindo para a cura do organismo. Desse modo, a aplicabilidade do ozônio é recomendada na prevenção e tratamento das osteonecroses dos maxilares e trabalhos futuros para investigar o benefício dessa tecnologia e sua aplicabilidade em diversas patologias, bem como estabelecimento de protocolos, concentrações e vias de administração são necessários.

\section{Referências}

Agrillo, A., Filiaci, F., Ramieri, V., Riccardi, E., Quarato, D., Rinna, C., \& Ungari, C. (2012). Bisphosphonate-related osteonecrosis of the jaw (BRONJ): 5 year experience in the treatment of 131 cases with ozone therapy. Eur Rev Med Pharmacol Sci, 16(12), 1741-7.

Agrillo, A., Sassano, P., Rinna, C., Priore, P., \& Iannetti, G. (2007a). Ozone therapy in extractive surgery on patients treated with bisphosphonates. Journal of Craniofacial Surgery, 18(5), 1068-1070.

Agrillo, A., Ungari, C., Filiaci, F., Priore, P., \& Iannetti, G. (2007b). Ozone therapy in the treatment of avascular bisphosphonate-related jaw osteonecrosis. Journal of Craniofacial Surgery, 18(5), 1071-1075.

Akdeniz, S. S., Beyler, E., Korkmaz, Y., Yurtcu, E., Ates, U., Araz, K., \& Torun, O. Y. (2018). The effects of ozone application on genotoxic damage and wound healing in bisphosphonate-applied human gingival fibroblast cells. Clinical oral investigations, 22(2), 867-873. 
Ali, M. A. A., Soliman, H. Á., Nizami, K., Chandra, R., Trukral, H., \& Phukan, A. H. (2018). Ozone therapy in dentistry: a literature review. Ejbps, 5 (2), $258-$ 261.

Alves, W. N. S. (2017). Ozonioterapia em caso de osteonecrose avançada associada a bisfosfonato oral em paciente com osteoporose: relato de caso. Monografia: Graduação em odontologia. Brasília: Faculdade de Ciências da Saúde da Universidade de Brasília.

Batinjan, G., Zore, I. F., Vuletić, M., \& Rupić, I. (2014). The use of ozone in the prevention of osteoradionecrosis of the jaw. Saudi medical journal, 35(10), 1260 .

Botelho, L. L. R., de Almeida Cunha, C. C., \& Macedo, M. (2011). O método da revisão integrativa nos estudos organizacionais. Gestão e sociedade, 5(11), $121-136$

Ellis, E. I. I. I. Tratamento do Paciente Submetido à Radioterapia ou à Quimioterapia. In: Hupp, J. R., Ellis, E. T., \& MR, C. (2015). Oral e maxilofacial contemporânea (p. 980). Elsevier.

Ferreira, M. B. (2011). Efeito na reparação óssea periapical da ozonioterapia como coadjuvante ao tratamento endodôntico. Estudo clínicoradiográfico (Doctoral dissertation, Universidade de São Paulo).

Ferreira, S., Mariano, R. C., Garcia Júnior, I. R., \& Pellizzer, E. P. (2013). Ozonioterapia no controle da infecção em cirurgia oral. Revista Odontológica de Araçatuba, 36-36.

Fliefel, R., Tröltzsch, M., Kühnisch, J., Ehrenfeld, M., \& Otto, S. (2015). Treatment strategies and outcomes of bisphosphonate-related osteonecrosis of the jaw (BRONJ) with characterization of patients: a systematic review. International journal of oral and maxillofacial surgery, 44(5), 568-585.

Garg, R., \& Tandon, S. (2009). Ozone: A new face of dentistry. Int J Dent Sci, 7(2).

Gopalakrishnan, S., \& Parthiban, S. (2012). Ozone-a new revolution in dentistry. J Bio Innov, 1(3), 58-69.

Gupta, G., \& Mansi, B. (2012). Ozone therapy in periodontics. Journal of medicine and life, 5(1), 59.

Huth, K. C., Jakob, F. M., Saugel, B., Cappello, C., Paschos, E., Hollweck, R., \& Brand, K. (2006). Effect of ozone on oral cells compared with established antimicrobials. European journal of oral sciences, 114(5), 435-440.

Huth, K. C., Quirling, M., Maier, S., Kamereck, K., Alkhayer, M., Paschos, E., \& Hickel, R. (2009). Effectiveness of ozone ag ainst endodontopathogenic microorganisms in a root canal biofilm model. International endodontic journal, 42(1), 3-13.

Huth, K. C., Paschos, E., Brand, K., \& Hickel, R. (2005). Effect of ozone on non-cavitated fissure carious lesions in permanent molars. A controlled prospective clinical study. American journal of dentistry, 18(4), 223-228.

Ikebe, T. (2013). Pathophysiology of BRONJ: drug-related osteoclastic disease of the jaw. Oral Science International, 10(1), 1-8.

Díaz Luis, J., Macías Abraham, C., \& Menéndez Cepero, S. (2013). Efecto modulador de la ozonoterapia sobre la actividad del sistema inmune. Revista Cubana de Hematología, Inmunología y Hemoterapia, 29(2), 143-153.

Naik, S. V., Rajeshwari, K., Kohli, S., Zohabhasan, S., \& Bhatia, S. (2016). Suppl-1, M7: Ozone-A Biological Therapy in Dentistry-Reality or Myth? The open dentistry journal, 10, 196.

Nesi, A. K. (2018). Ozonioterapia: o uso do ozônio na odontologia. 24f. Trabalho de conclusão de curso - Bacharelado em Odontologia, Centro Universitário São Lucas, Porto Velho, 2018.

Nogales, C. G., Ferreira, M. B., Campos, F. U. F., Siqueira, M., \& Macedo, S. B. (2020). Ozone therapy: adjuvant to endodontic treatment in a subluxation case-case report. Revista Española de Ozonoterapia, 10(1), 171-177.

Nogales, C. G., Ferreira, M. B., Montemor, A. F., Rodrigues, M. F. D. A., Lage-Marques, J. L., \& Antoniazzi, J. H. (2016). Ozone therapy as an adjuvant for endondontic protocols: microbiological-ex vivo study and citotoxicity analyses. Journal of Applied Oral Science, 24, 607-613.

Nogales, C. G., Ferrari, P. H., Kantorovich, E. O., \& Lage-Marques, J. L. (2008). Ozone therapy in medicine and dentistry. J Contemp Dent Pract, $9(4), 75-84$.

Nogales, C. G. (2011). Parâmetros da ação antimicrobiana e da citotoxicidade do ozônio para aplicação na Endodontia [dissertação]. São Paulo: Faculdade de Odontologia da Universidade de São Paulo.

Omolehinwa, T. T., \& Akintoye, S. O. (2016). Chemical and radiation-associated jaw lesions. Dental Clinics, 60(1), 265-277.

Pulga, A. (2018). Oxygen-ozone therapy in dentistry: current applications and future prospects. Ozone Therapy, 3(3).

Reddy, S., Reddy, N., Dinapadu, S., Reddy, M., \& Pasari, S. (2013). Role of ozone therapy in minimal intervention dentistry and endodontics-a review. Journal of international oral health: JIOH, 5(3), 102.

Ribeiro, G. H., Chrun, E. S., Dutra, K. L., Daniel, F. I., \& Grando, L. J. (2018). Osteonecrosis of the jaws: a review and update in etiology and treatment ir. Brazilian journal of otorhinolaryngology, 84, 102-108.

Ripamonti, C. I., Cislaghi, E., Mariani, L., \& Maniezzo, M. (2011). Efficacy and safety of medical ozone (O3) delivered in oil suspension applications for the treatment of osteonecrosis of the jaw in patients with bone metastases treated with bisphosphonates: Preliminary results of a phase I-II study. Oral oncology, 47(3), 185-190. 
Research, Society and Development, v. 10, n. 11, e72101119276, 2021

(CC BY 4.0) | ISSN 2525-3409 | DOI: http://dx.doi.org/10.33448/rsd-v10i11.19276

Ripamonti, C. I., Maniezzo, M., Boldini, S., Pessi, M. A., Mariani, L., \& Cislaghi, E. (2012). Efficacy and tolerability of medical ozone gas insufflations in patients with osteonecrosis of the jaw treated with bisphosphonates-Preliminary data: medical ozone gas insufflation in treating ONJ lesions. Journal of bone oncology, 1(3), 81-87.

Rodrigues, R. C. D. S. (2016). Ozonioterapia em paciente com osteonecrose mandibular: relato de caso.

Sabbah, F., Nogales, C. G., Zaremski, E., \& Martinez-Sanchez, G. (2018). Ozone therapy in Dentistry-Where we are and where we are going to?. Ozone Therapy Global Journal, 8(1), 37-63.

Smith, N. L., Wilson, A. L., Gandhi, J., Vatsia, S., \& Khan, S. A. (2017). Ozone therapy: an overview of pharmacodynamics, current research, and clinical utility. Medical gas research, 7(3), 212.

Srikanth, A., Sathish, M., \& Harsha, A. V. S. (2013). Application of ozone in the treatment of periodontal disease. Journal of pharmacy \& bioallied sciences, 5(Suppl 1), S89.

Suh, Y., Patel, S., Kaitlyn, R., Gandhi, J., Joshi, G., Smith, N. L., \& Khan, S. A. (2019). Clinical utility of ozone therapy in dental and oral medicine. Medical gas research, 9(3), 163.

Tiwari, S., Avinash, A., Katiyar, S., Iyer, A. A., \& Jain, S. (2017). Dental applications of ozone therapy: A review of literature. The Saudi Journal for Dental Research, 8(1-2), 105-111. 